\title{
Update on multi-variable parametric cost models for ground and space telescopes
}

\author{
H. Philip Stahl ${ }^{\mathrm{a}}$, Todd Henrichs ${ }^{\mathrm{b}}$, Alexander Luedtke ${ }^{\mathrm{c}}$ and Miranda $\mathrm{West}^{\mathrm{d}}$ \\ ${ }^{a}$ NASA MSFC, Huntsville, AL 35821; \\ ${ }^{\mathrm{b}}$ Department of Mathematical Sciences, Middle Tennessee State University; \\ ${ }^{\mathrm{c}}$ Division of Applied Mathematics, Brown University; and \\ ${ }^{\mathrm{d} D e p a r t m e n t}$ of Mathematics, University of Texas at Austin
}

\begin{abstract}
Parametric cost models can be used by designers and project managers to perform relative cost comparisons between major architectural cost drivers and allow high-level design trades; enable cost-benefit analysis for technology development investment; and, provide a basis for estimating total project cost between related concepts. This paper reports on recent revisions and improvements to our ground telescope cost model and refinements of our understanding of space telescope cost models. One interesting observation is that while space telescopes are 50X to $100 \mathrm{X}$ more expensive than ground telescopes, their respective scaling relationships are similar. Another interesting speculation is that the role of technology development may be different between ground and space telescopes. For ground telescopes, the data indicates that technology development tends to reduce cost by approximately $50 \%$ every 20 years. But for space telescopes, there appears to be no such cost reduction because we do not tend to re-fly similar systems. Thus, instead of reducing cost, 20 years of technology development may be required to enable a doubling of space telescope capability. Other findings include: mass should not be used to estimate cost; spacecraft and science instrument costs account for approximately $50 \%$ of total mission cost; and, integration and testing accounts for only about $10 \%$ of total mission cost.
\end{abstract}

Keywords: Space Telescope Cost Model, Parametric Cost Model

\section{INTRODUCTION}

Parametric cost models for space telescopes provide several benefits to designers and space system project managers. They identify major architectural cost drivers and allow high-level design trades; enable cost-benefit analysis for technology development investment; and, they provide a basis for estimating total project cost. Over the past eight years, we have published several papers on cost models for both ground and space based optical telescope assemblies (OTA), where an OTA is defined as the primary mirror, secondary mirror, auxiliary optics, and support structure. ${ }^{[1-10]}$ These papers fully discuss our data collection methodology and cost model creation process. A discussion of that methodology and statistical analysis process will not be repeated in this paper. This paper provides a summary update of our recent progress in developing multi-variable parametric cost models for both ground and space telescopes.

\section{GROUND OPTICAL TELESCOPE ASSEMBLY COST MODEL}

In 2005, Stahl et al. published a single variable which estimated cost as a function of primary mirror diameter and a multivariable parametric cost model for ground telescopes which estimated cost as a function of primary mirror diameter, diffraction-limited wavelength, and year of development. ${ }^{[2]}$ The multi-variable model also included a factor for primary mirror segmentation and/or duplication. While the original multivariable model is still relevant an error was found and corrected in the single variable model. ${ }^{[10]}$ The single variable model published in 2005 is:

$$
\text { Ground OTA Cost } \propto \text { Diameter }{ }^{1.8}
$$

The corrected model published in 2012 is:

$$
\text { Ground OTA Cost } \sim \$ 2 \mathrm{M} \text { x Diameter }{ }^{1.4}
$$

As discussed in Luedtke and Stahl, 2012, the reason that the diameter exponent changed is because the leading multiplier was excluded from the regression. The reason it was excluded is because cost models are inherently comparative, i.e. they should not be used to actually attempt to predict the cost of a given specific telescope architecture, but rather they should be used to compare the relative costs of two related telescopes. But, the effect of excluding the leading multiplier from the regression was to force the cost estimate of small aperture telescopes towards 1; which required a 'steeper' cost 
curve with diameter. Also, by omitting the leading multiplier, the regression was not independent to units, i.e. a different exponent would have been returned if the regression had been done in centimeters instead of meters. By including the leading multiplier, a 'flatter' cost curve with diameter that is independent of units was obtained.

Another reason that the 2005 paper published the single variable model without the multiplier is because the coefficient was similar with that of the multi-variable model, but in fact it should not have matched. The reason why the single variable diameter exponent should be lower than the multivariable diameter exponent is because of the effect of year of development. Year of development and aperture diameter are positively correlated $(\mathrm{r}=0.55, \mathrm{p}=0.03)$, a fact which makes sense because one tends to build the largest telescope that can be afforded at any given time. Because technology advancement tends to reduce the cost to fabricate a telescope of a given aperture diameter, when year of development is implicitly included in the single variable model, it results in a smaller diameter exponent. But, when year of telescope development is made explicit as a cost variable independent of aperture diameter, it results in a larger diameter exponent.

The Stahl 2005 multivariable model had a diameter exponent of 1.8:

$$
\begin{gathered}
\text { Ground OTA Cost } \sim 0.7 \text { SF D } D^{1.8} \lambda^{-0.5} \mathrm{e}^{-0.04(\mathrm{Y})} \\
\left(\mathrm{R}^{2}=95 \%, \text { adjusted } \mathrm{R}^{2}=94 \%, \mathrm{SPE}=35 \%\right)
\end{gathered}
$$

Where:

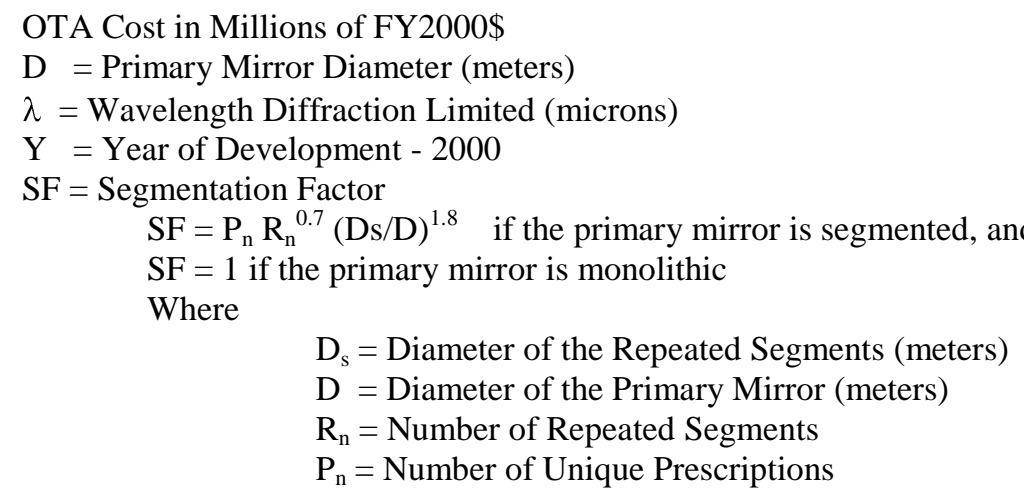

A key element of the multi-variable ground telescope mode is the Segmentation Factor. The purpose of this factor is to include cost reduction from learning while making duplicate primary mirror segments (such as for Keck or HET) or whole primary mirrors for arrays of telescopes (such as for VLT or CARA). The data base used to derive the above result contained only 5 segmented monolithic or sparse aperture telescopes. The 0.7 exponent is an $80 \%$ learning curve (where each successive unit is $80 \%$ the cost of the previous unit). And, in all cases the duplicated segments or complete OTAs were assumed to be identical. Therefore, an assumption was made that learning was confined to a given prescription or implementation. However, results from the JWST primary mirror assembly segments (PMSAs) indicate that learning transfers across prescriptions (Figure 1).
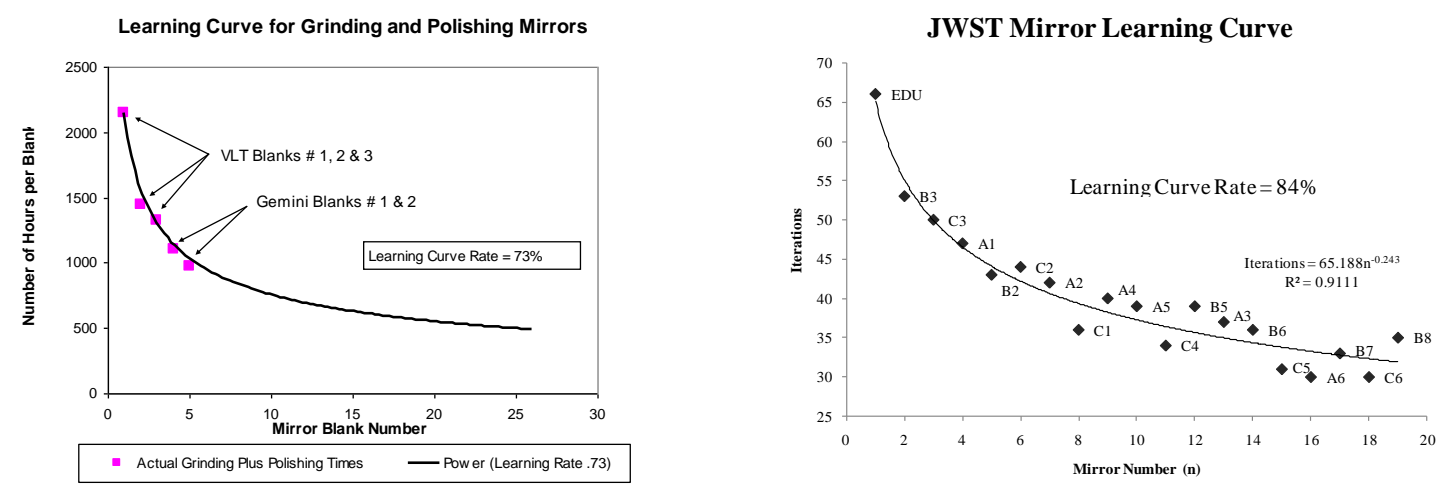

Figure 1: Learning Curve for fabricating 5 8-meter primary mirrors for VLT \& Gemini is 73\%; Learning curve for fabricating 19 1.5-meter segments for JWST is $84 \%$ (private communication, Ben Gallagher). 
Given the JWST results, Luedtke and Stahl 2012, published a new ground telescope cost model. The model is derived from a data set of from only 5 segmented or sparse aperture telescopes and assumes that learning transfers across prescriptions:

$$
\begin{gathered}
\text { Ground OTA Cost } \sim 0.8 \mathrm{SF} \mathrm{D}^{1.7} \lambda^{-0.7} \mathrm{e}^{-0.04(\mathrm{Y})} \\
\left(\mathrm{R}^{2}=91 \%, \text { adjusted } \mathrm{R}^{2}=88 \%, \mathrm{SPE}=37 \%\right)
\end{gathered}
$$

where $\mathrm{SF}=\left[\mathrm{S}^{0.7}(\mathrm{Ds} / \mathrm{D})^{1.7}\right]$ and $\mathrm{S}$ represents the total number of segments. While the duplication and diameter exponents are nearly identical, the wavelength exponent is significantly different. However, given the size and similarity of the current data base, the new - 0.7 exponent may or may not be more correct than the old -0.5 exponent. The problem is one of insufficient wavelength diversity in the data base. While multiple authors have given rules of thumb for how cost varies with wavelength, only Meinel's papers contain quantitative data. ${ }^{[4]}$ A comparison of optical and radio wavelength telescopes indicates approximately a $100 \mathrm{X}$ to $1000 \mathrm{X}$ difference in cost. ${ }^{[11,12]}$ Assuming that an optical telescope operates at a wavelength of 1 micrometer and that radio telescopes operate from $10 \mathrm{~mm}$ to 10 meters, an exponent of -0.5 implies that a radio telescope will cost $100 \mathrm{X}$ to $3000 \mathrm{X}$ less while an exponent of -0.7 implies a 600 to $80,000 \mathrm{X}$ cost reduction. Similarly, a comparison of optical and submillimeter telescopes indicates a 10X cost difference ${ }^{[13]}$ Assuming that the submillimeter wavelength is 100 micrometers to $1 \mathrm{~mm}$, then a -0.5 exponent indicates a $10 \mathrm{X}$ to $30 \mathrm{X}$ cost reduction while a -0.7 exponent indicates a 25 to $125 \mathrm{X}$ reduction.

\section{SPACE OPTICAL TELESCOPE ASSEMBLY COST MODEL}

While some of the space telescope data base has changed slightly since 2011, there is no change to any of the model regressions. However, we continue to refine our interpretations of the data and try to clarify mis-conceptions.

\subsection{Optical Telescope Assembly Cost as a percentage of Total Mission Cost}

During the past year, this author has been in several meetings where some have asserted, quite forcefully, that one must reduce the size of a telescope aperture to make a given mission affordable. Or, that integration and test (I\&T) accounts for $60 \%$ of the total mission cost and that all one needs to do is reduce the need for I\&T. The problem is that the data does not support these assertions. Based on an analysis of detailed work breakdown structures for 7 of the 14 free flying UVOIR space telescope missions in the data base for which we have both OTA and Total Mission cost, the average OTA cost is only $12 \%$ of total mission cost (Figure 2). For these missions, the spacecraft and science instruments account for half of the total mission cost, and the OTA cost is comparable with Program Management and Systems Engineering cost. It must be stated that the $12 \%$ OTA cost is an average. The actual costs of OTA as a function of Total, for all 14 missions for which we have data, varies from 2 to $20 \%$ (Figure 3). Based on this analysis, one might conclude that the best way to reduce total mission cost is to reduce the cost (and or capability) of the Science Instruments or Spacecraft. But, maybe the better question to ask is What is right balance of cost between major subsystems? And, which subsystem's cost is out of proportion?

Furthermore, Observatory level I\&T cost is only $10 \%$ of total mission cost. However, in this accounting, mission I\&T cost excludes sub-system level I\&T cost. Given that sub-system I\&T costs might be 10 to $25 \%$ of their individual costs, it is plausible that total I\&T costs might be as large as $25 \%$ of total mission cost but certainly not $60 \%$.

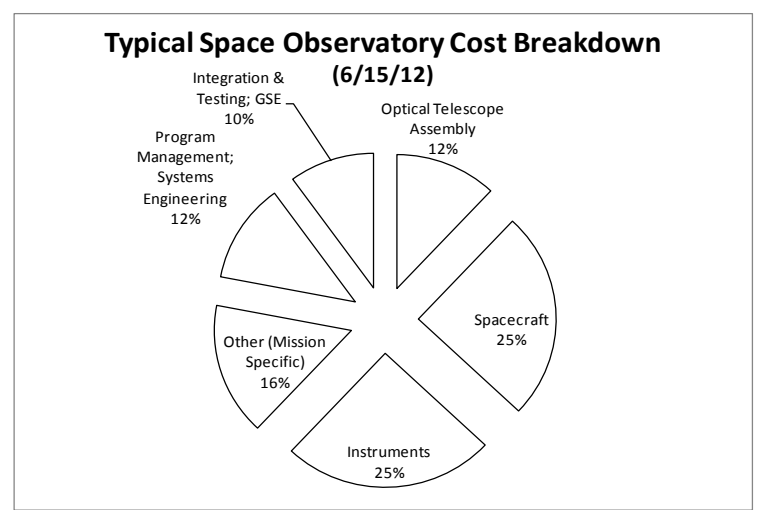

Figure 2: Average Percentage of Total Mission Cost for Major Cost Elements and/or Sub-Systems.

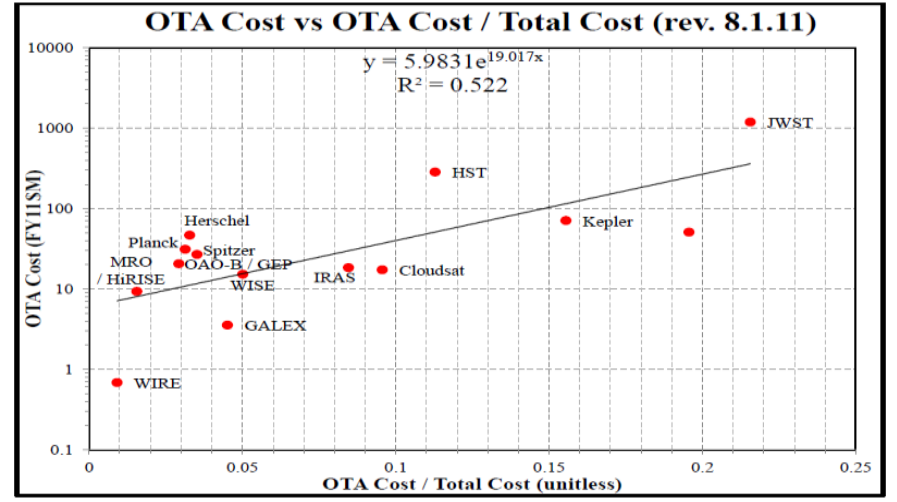

Figure 3: OTA Cost as a function of Total Mission Cost 


\subsection{Optical Telescope Assembly Cost as a function of Mass}

Many believe that mass is the single most important cost driver for space telescopes. They assert that to reduce cost, all one need do is to reduce mass. But, it is simply not true. A comparison of JWST and HST proves the point. If mass determined cost, then the JWST OTA would cost approximately the same as the HST OTA instead of twice as much. And, if mass determined cost, then the total mission cost of JWST would be half the total mission cost of HST instead of the reverse (Figure 4).
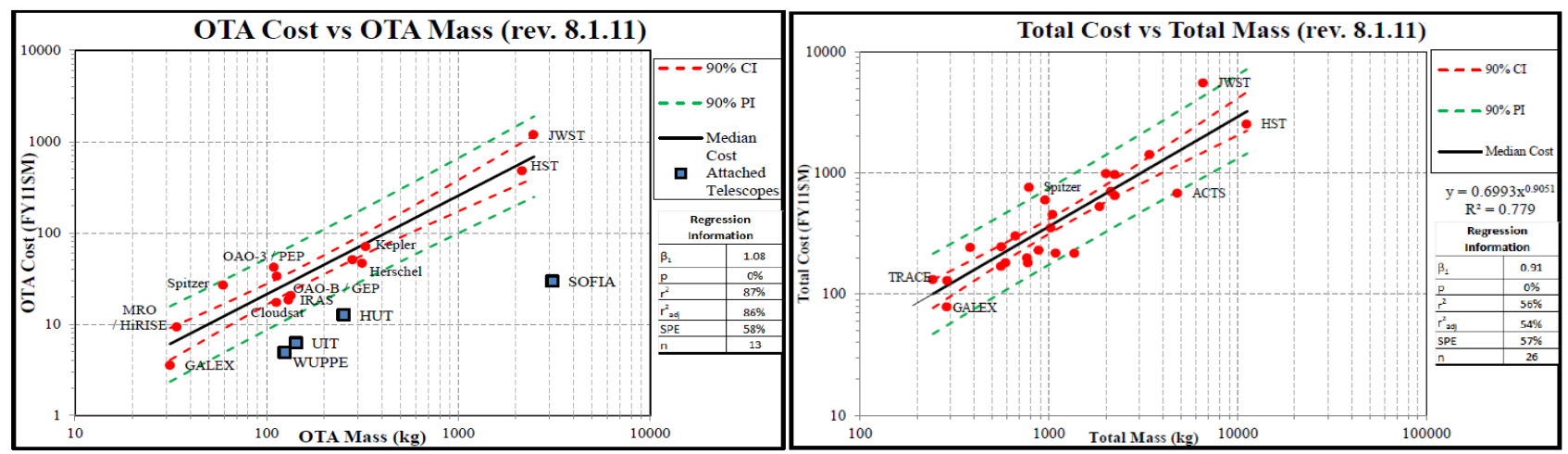

Figure 4: Mass of the JWST and HST OTAs are nearly identical, but the cost of the JWST OTA is twice the cost of the HST OTA; Total mass of HST is nearly twice that of JWST, but the cost of JWST is nearly twice that of HST.

What is true is that space missions are constrained by the mass capacity of their launch vehicle. All space missions are designed to a constrained mass budget. Thus, mass is not a free variable. And, it is a fact that ground telescopes cost less than space telescopes. What is interesting is that all ground telescopes have approximately the same cost per $\mathrm{kg}$. And, all space telescopes also have approximately the same cost per $\mathrm{kg}$ (Figure 5). But the cost per $\mathrm{kg}$ for ground and space differ by 1000X. Additionally, SOFIA which is attached to a 747 airframe is $15 \mathrm{X}$ less expensive per $\mathrm{kg}$ than free-flying space telescopes. And, space telescopes which were attached to the Space Shuttle were $2 \mathrm{X}$ less expensive than free-flying space telescopes. One conclusion is that design rules drive OTA cost more than mass: complex, smart mass with tight design rules costs more per $\mathrm{kg}$ than simple, dumb mass with generous design rules. Thus, the best way to reduce cost is to change the design rules by using larger capacity launch vehicles and increasing the mass margins.

\subsection{Single Variable Space Optical Telescope Assembly Cost Model}

While the single variable space OTA model is unchanged, it is interesting to note that its diameter exponent is now identical to the ground OTA model.

$$
\begin{gathered}
\text { Space OTA Cost } \sim \$ 100 \mathrm{MD}^{1.4}\left(N=15 ; r^{2}=82 \% ; \text { SPE }=123\right) \\
\text { Ground OTA Cost } \sim \$ 2 \mathrm{M} \mathrm{D}^{1.4}
\end{gathered}
$$

Based on 15 free-flying space telescopes, the aperture diameter model accounts for $82 \%$ of the cost variation in the real data and it is noisy. Therefore, a multi-variable model in indicated.

Space Telescopes \& Instrumentation 2012: Optical, Infrared, and Millimeter Wave, SPIE Proceedings 8442, Amsterdam 2012 


\subsection{Multivariable Space Optical Telescope Assembly Cost Models}

Given that the single variable aperture model accounts for only $82 \%$ of the actual OTA cost variation and is somewhat noisy, it is necessary to look at multi-variable models. To develop a multi-variable model, we regress cost against diameter and candidate second variables (Figure 6). A good two variable model is one where the second variable

\begin{tabular}{|c|c|c|c|c|c|c|c|c|}
\hline \multicolumn{2}{|c|}{ rev. 8.1.11 } & \multicolumn{7}{|c|}{ OTA Cost vs Aperture Diameter and V2 } \\
\hline & 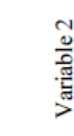 & 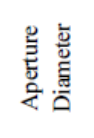 & 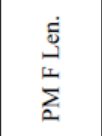 & 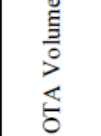 & o & 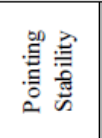 & 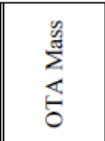 & 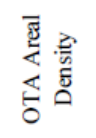 \\
\hline Diam & $p$-value & \begin{tabular}{|l|l|}
1.42 & 0.00 \\
\end{tabular} & \begin{tabular}{|l|l|l|}
0.73 & 0.19 \\
\end{tabular} & \begin{tabular}{|l|l|l|}
-1.28 & 0.38 \\
\end{tabular} & \begin{tabular}{|l|l|}
1.26 & 0.02 \\
\end{tabular} & \begin{tabular}{|l|l|}
1.64 & 0.01 \\
\end{tabular} & \begin{tabular}{|l|l|}
0.02 \\
\end{tabular} & \begin{tabular}{|l|l|}
2.05 & 0.00 \\
\end{tabular} \\
\hline Var. 2 & $p$-value & - & \begin{tabular}{|l|l|}
1.00 & 0.06 \\
\end{tabular} & \begin{tabular}{|l|l|}
1.00 & 0.06 \\
\end{tabular} & \multirow{2}{*}{$\begin{array}{c}0.00 \\
4 \%\end{array}$} & \multirow{2}{*}{$\frac{-0.21 \mid 0.32}{95 \%}$} & \begin{tabular}{|l|l|}
1.07 & 0.00 \\
\end{tabular} & \begin{tabular}{|l|l|}
1.01 & 0.00 \\
\end{tabular} \\
\hline \multicolumn{2}{|c|}{ Adjusted $\mathrm{r}^{2}$} & $81 \%$ & $93 \%$ & $93 \%$ & & & $85 \%$ & $84 \%$ \\
\hline \multicolumn{2}{|c|}{ SPE } & $123 \%$ & $84 \%$ & $84 \%$ & $142 \%$ & $66 \%$ & $58 \%$ & $54 \%$ \\
\hline \multicolumn{2}{|c|}{$\mathrm{n}$} & 15 & \multirow{2}{*}{$\frac{11}{\text { Yes }}$} & \multirow{2}{*}{11} & 12 & 8 & $\mathrm{y}$ & 12 \\
\hline \multicolumn{2}{|c|}{ Multicollinearity? } & N/A & & & No & No & Yes & No \\
\hline \multicolumn{2}{|c|}{ 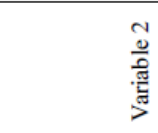 } & 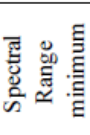 & & 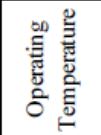 & 莺 & 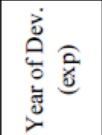 & 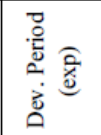 & 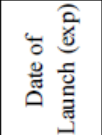 \\
\hline Diam & $p$-value & \begin{tabular}{|l|l|}
1.62 & 0.00 \\
\end{tabular} & \begin{tabular}{|l|l|}
1.54 & 0.00 \\
\end{tabular} & \begin{tabular}{|l|l|}
1.49 & 0.00 \\
\end{tabular} & \begin{tabular}{|l|l|}
0.83 & 0.02 \\
\end{tabular} & \begin{tabular}{|l|l|}
1.45 & 0.00 \\
\end{tabular} & \begin{tabular}{|l|l|}
1.14 & 0.04 \\
\end{tabular} & \begin{tabular}{|l|l|}
1.46 & 0.00 \\
\end{tabular} \\
\hline Var. 2 & $p$-value & \begin{tabular}{|l|l|}
-0.18 & 0.02 \\
\end{tabular} & \begin{tabular}{|l|l|}
-0.22 & 0.02 \\
\end{tabular} & \begin{tabular}{l|l|l|}
-0.08 & 0.64 \\
\end{tabular} & \begin{tabular}{|l|l|}
0.01 & 0.01 \\
\end{tabular} & \begin{tabular}{l|l}
-0.01 & 0.46 \\
\end{tabular} & \begin{tabular}{|l|l|l|}
0.01 & 0.17 \\
\end{tabular} & \begin{tabular}{|l|l|}
-0.01 & 0.7 \\
\end{tabular} \\
\hline Adjus & $\operatorname{sted~} r^{2}$ & $96 \%$ & $98 \%$ & $81 \%$ & $99 \%$ & $84 \%$ & $91 \%$ & $82 \%$ \\
\hline & $\overline{\mathrm{PE}}$ & $74 \%$ & $60 \%$ & $136 \%$ & $71 \%$ & $124 \%$ & $128 \%$ & $120 \%$ \\
\hline & $\mathrm{n}$ & 15 & 12 & 14 & 15 & 14 & 13 & 15 \\
\hline Itic & & No & Nc & No & No & & No & No \\
\hline
\end{tabular}

Figure 6: 2 Variable OTA Cost Regression Summary exponent is significant (has a p-value less than 0.1 ), its addition does not affect the significance of the Diameter exponent, and the combination of the two increases adjusted-r ${ }^{2}$ and decreases SPE. Also, the second variable must not be collinear with the aperture diameter. The three variables which are collinear with aperture diameter are primary mirror focal length, OTA volume and OTA mass. In each case, their exponent is significant, but their addition invalidates the aperture diameter exponent. Of these three variables, OTA volume might actually be a better cost estimating parameter than diameter. But, it is much harder to quantify. An interesting observation is that Year of Development (YOD) or year of launch is not significant. Given that YOD is important for ground telescopes, this is a most curious result. Why does technology advances tend to reduce cost as a function of time for ground telescopes but not for space telescopes. One explanation is that unlike ground telescopes, where each new telescope is a variation upon the theme, most space telescopes are not. Most space telescopes are unique, one of a kind designs which require the invention of new technology just to exist. Technology advance is most effective when making a second or duplicate system.

Ignoring areal density because diameter is in both variables, the best second variables which yield a significant exponent without invalidating the diameter exponent are spectral range, diffraction limited wavelength and design life. While design life has the highest adjusted- $\mathrm{r}^{2}$ its exponent is has no value. Thus, the best two variable cost model is:

$$
\text { OTA Cost } \sim \operatorname{Dia}^{1.6} \lambda^{-0.2} \quad\left(N=12, r^{2}=98 \% ; S P E=60 \%\right)
$$

Repeating the regression analysis for a third variable yields:

$$
\text { OTA Cost } \sim \mathrm{D}^{1.7} \lambda^{-0.3} \mathrm{~T}^{-0.25} \quad\left(N=11, r^{2}=96 \% ; \text { SPE }=54 \%\right)
$$

It is obvious why temperature is the next variable. Just as aperture diameter and year of development are inversely correlated for ground telescopes, wavelength and temperature are negatively correlated for space telescopes. For space telescopes, the longer the operating wavelength, the lower the operating temperature. Two additional observations, the sign and magnitudes of the wavelength and temperature exponents tend to balance each other; and adding the second and third parameters has moved the diameter exponent closer to the ground model.

\section{CONCLUSIONS}

Parametric cost models provide several benefits to designers and space system project managers. They identify major architectural cost drivers and allow high-level design trades; enable cost-benefit analysis for technology development investment; and, they provide a basis for estimating total project cost. As part of our continuing research, we have revised and improved our ground telescope cost model and refined our understanding of space telescope cost models.

Parametric Cost Models should not be used to predict the cost of a specific architecture, but rather estimate the cost difference between similar architectures. For example, for ground based telescopes aperture diameter is the most significant cost driver and technology development tends to reduce cost by $\sim 50 \%$ every 20 years. Also, longer wavelength telescopes are less expensive to build than shorter wavelength telescopes. Because all ground telescopes operate at approximately the same temperature, there is no statistical cost dependency for operating temperature. For space telescopes, aperture diameter is also the most significant cost driver, and, while space telescopes cost more than 
ground telescopes, the relative scaling of cost with aperture diameter is similar for the two classes of telescopes. One explanation for why space telescopes cost more than ground telescopes is the design rules, i.e. the requirement to design space telescopes to a constrained mass budget with tight safety margins. Mass is not a good (or maybe even valid) cost estimating parameter. While in our data base larger aperture telescopes always cost more than smaller aperture telescopes, this relationship does not always hold true for mass. Additionally, for space telescopes, we are not finding a statistically significant cost reduction with year of development. One explanation may be because space telescopes tend to be unique, one-of-a-kind systems which rather than taking advantage of technology development to reduce their costs, actually take advantage of technology development to enable their existence. Therefore, instead of reducing cost by $\sim 50 \%$ every 20 years, maybe 20 years of technology development is required to enable a doubling of a given mission capability. For both ground and space telescopes, when manufacturing duplicative components, the data indicates $\sim 80 \%$ learning curve cost reduction.

Finally, analysis of detailed work breakdown structures for 7 space missions indicates that, on average, the optical telescope assembly is approximately $12 \%$ of total mission cost. By comparison, spacecraft and science instrument costs account for approximately $50 \%$ of total mission cost. And, depending upon how the costs are accounted, integration and testing costs are only $10 \%$ to maybe $25 \%$ of total mission cost.

\section{REFERENCES}

[1] Stahl, H. Philip, Ginger Holmes Rowell, Gayle Reese and Alicia Byberg, "Ground-Based Telescope Parametric Cost Model”, SPIE Porceedings, Vol.5497, p.173, 2004.

[2] Stahl, H. Philip, Ginger Holmes Rowell, Gayle Reese, and Alicia Byberg, "Multivariable parametric cost model for ground optical telescope assembly", Optical Engineering. Vol. 44, 083001 (2005)

[3] Stahl, H. Philip, Frank A. Prince, Christian Smart, Kyle Stephens and Todd Henrichs, "Preliminary Cost Model for Space Telescopes", SPIE Proceedings, Vol.7436, 2009.

[4] Stahl, H. Philip, "Survey of Cost Models for Space Telescopes", Optical Engineering, Vol.49, No.05, 2010, DOI:10.1117/1.3430603

[5] Stahl, H. Philip, Kyle Stephens, Todd Henrichs, Christian Smart, and Frank A. Prince, "Single Variable Parametric Cost Models for Space Telescopes", Optical Engineering Vol.49, No.07, 2010, DOI:10.1117/1.3456582

[6] Stahl, H. Philip, and Todd Henrichs, "Preliminary Multi-Variable Cost Model for Space Telescopes", $\underline{\text { SPIE }}$ Proceedings, Vol.7731, 2010, DOI:10.1117/12.856214

[7] Stahl, H. Philip, Todd Henrichs, Courtnay Dollinger, "Parametric Cost Models for Space Telescopes", International Conference on Space Optics, Rhodes Greece, Oct 2010.

[8] Stahl, H. Philip, and Todd Henrichs, "Cost modeling for space optical telescope assemblies", SPIE Proceedings, Vol.8011, 22nd Congress of the International Commission for Optics: Light for the Development of the World, 2011

[9] Stahl, H. Philip, Todd Henrichs, Alexander Luedtke, and Miranda West, "Update on parametric cost models for space telescopes", SPIE Proceedings, Vol.8146, 2011, DOI:10.1117/12.894085

[10] Luedtke, Alexander and H. Philip Stahl, "Commentary on Multivariable Parametric Cost Model for Ground Optical Telescope Assembly”, Optical Engineering Vol.51, 059701 (2012), DOI:10.1117/1.OE.51.5.059701

[11] Meinel, A. B., "Cost-scaling Laws Applicable to Very Large Telescopes." Optical Engineering. Vol. 18, No. 6. Pages 645-647. November/December, 1979.

[12] Meinel A. B., "Cost Relationships for Nonconventional Telescope Structural Configurations." Journal Optical Society of America. Vol. 2, No 1, pages 14-20. January 1981.

[13] Meinel, Aden Baker and Marjorie Pettit Meinel, "Optical phased array configuration for an extremely large telescope", Applied Optics, Vol 43, No 3, pp.601-607, 2004. 